(c) American Dairy Science Association, 2005.

\title{
Genetic Progress in Multistage Dairy Cattle Breeding Schemes Using Genetic Markers
}

\author{
C. Schrooten, ${ }^{1,2}$ H. Bovenhuis, ${ }^{1}$ J. A. M. van Arendonk, ${ }^{1}$ and P. Bijma ${ }^{1}$ \\ ${ }^{1}$ Animal Breeding and Genetics Group, Wageningen University, PO Box 338, \\ $6700 \mathrm{AH}$ Wageningen, The Netherlands \\ ${ }^{2}$ Holland Genetics, CR Delta, PO Box 5073, 6802 EB Arnhem, The Netherlands
}

\begin{abstract}
The aim of this paper was to explore general characteristics of multistage breeding schemes and to evaluate multistage dairy cattle breeding schemes that use information on quantitative trait loci (QTL). Evaluation was either for additional genetic response or for reduction in number of progeny-tested bulls while maintaining the same response. The reduction in response in multistage breeding schemes relative to comparable single-stage breeding schemes (i.e., with the same overall selection intensity and the same amount of information in the final stage of selection) depended on the overall selection intensity, the selection intensity in the various stages of the breeding scheme, and the ratio of the accuracies of selection in the various stages of the breeding scheme. When overall selection intensity was constant, reduction in response increased with increasing selection intensity in the first stage. The decrease in response was highest in schemes with lower overall selection intensity. Reduction in response was limited in schemes with low to average emphasis on first-stage selection, especially if the accuracy of selection in the first stage was relatively high compared with the accuracy in the final stage.

Closed nucleus breeding schemes in dairy cattle that use information on QTL were evaluated by deterministic simulation. In the base scheme, the selection index consisted of pedigree information and own performance (dams), or pedigree information and performance of 100 daughters (sires). In alternative breeding schemes, information on a QTL was accounted for by simulating an additional index trait. The fraction of the variance explained by the QTL determined the correlation between the additional index trait and the breeding goal trait. Response in progeny test schemes relative to a base breeding scheme without QTL information ranged from $+4.5 \%$ (QTL explaining $5 \%$ of the additive genetic variance) to $+21.2 \%$ (QTL explaining $50 \%$ of the addi-
\end{abstract}

Received June 19, 2004.

Accepted December 6, 2004.

Corresponding author: Chris Schrooten; e-mail: schrooten.c@hg.nl. tive genetic variance). A QTL explaining $5 \%$ of the additive genetic variance allowed a $35 \%$ reduction in the number of progeny tested bulls, while maintaining genetic response at the level of the base scheme. Genetic progress was up to $31.3 \%$ higher for schemes with increased embryo production and selection of embryos based on QTL information. The challenge for breeding organizations is to find the optimum breeding program with regard to additional genetic progress and additional (or reduced) cost.

(Key words: genetic evaluation, marker-assisted selection, quantitative trait locus, dairy cattle)

Abbreviation key: MAS = marker-assisted selection .

\section{INTRODUCTION}

In the past $10 \mathrm{yr}$, many efforts have been undertaken to detect genes underlying economically important traits in dairy cattle. Quantitative trait loci (i.e., chromosomal regions acting on a certain trait) have been localized without knowing the exact position or the mutation responsible for the effect. The first results were obtained for milk production traits (e.g., Georges et al., 1995), followed by results for conformation and functional traits (e.g., Ashwell et al., 1996). In some cases, the QTL have been fine-mapped, up to the point where the mutation responsible for the effect has been identified. This has been accomplished for monogenic traits like BLAD (bovine leukocyte adhesion deficiency; Shuster et al., 1992), and, more recently, for quantitative traits like milk production (Grisart et al., 2002; Blott et al., 2003).

To map QTL, genetic markers have been used. Markers for which linkage to specific QTL has been established can be used for marker-assisted selection (MAS). They give information on the inheritance of the QTL alleles and thus give information about the genetic potential of an animal. This information can be used to increase genetic progress, either by increasing the reliability of EBV, by reduction of the generation interval, or by increasing the selection intensity.

Current dairy cattle breeding schemes rely on progeny testing to assess the genetic value of bulls. Progeny 
testing increase the generation interval, but this shortcoming is compensated for by an increase in accuracy. Progeny testing would not be necessary if markers were available that explained a substantial fraction of the genetic variance. This ideal will not be realized in the near future, however, because breeding goals in dairy cattle consist of many traits. The MAS of sires will therefore most likely be applied as an initial selection step in a multistage selection setting. Marker information will increase the accuracy of selection, especially in the first stage. Application of MAS in the initial stage can be used to increase rate of gain through preselection or to decrease costs, by progeny testing of fewer young bulls while maintaining the rate of gain.

Soller and Beckmann (1982) suggested screening of sires to be progeny tested, based on genetic polymorphisms. Since then, various studies have evaluated a range of breeding schemes with MAS. Some of these studies looked at 2-stage selection schemes where candidate young bulls were selected based on pedigree and marker information (e.g., Kashi et al., 1990; Mackinnon and Georges, 1998; Spelman and Garrick, 1998). Another group of studies looked at selection in a single stage, combining all available information, including marker information (e.g., Meuwissen and van Arendonk, 1992; Meuwissen and Goddard, 1996), and allowing selection of parents across all age classes. Estimates for additional gain ranged from $-6 \%$ (Spelman and Garrick, 1997) to $+105 \%$ (Spelman et al., 1999). Profitability of MAS breeding schemes depends on several factors, such as the fraction of the genetic variation explained by the QTL, the frequencies of QTL alleles, the design of the breeding scheme, and the time horizon of evaluation.

Genotyping techniques can be applied to newborn animals, but they can also be applied to embryos (Bredbacka, 2001). Selection at the embryo stage offers additional opportunities, because the number of implanted embryos can be reduced, compared with a scheme based on selection of calves. To date, these opportunities have not been explored in detail.

Marker-assisted selection can be part of multistage selection: markers are typed early in life and used in the first stage of selection, for example, in combination with pedigree information. In the second stage, the remaining selection candidates are selected on a combination of first-stage information, and phenotypic information that has come available in the interim. The aim of this study was to evaluate dairy cattle breeding schemes regarding additional genetic response with MAS, and regarding possible changes to the structure of the breeding program when MAS is implemented in the breeding program.
Because dairy cattle breeding schemes are set up as multistage breeding schemes, and MAS is likely to be applied in such a multistage setting, this study examined 1) general aspects of multistage selection; and then addressed 2) the potential increase in genetic progress from MAS of newborn animals; 3) the possibilities to reduce the number of progeny tested young bulls in a MAS scheme while maintaining a constant rate of gain; and 4) the additional genetic progress from MAS in breeding schemes with increased embryo production and genotyping of embryos.

\section{MATERIALS AND METHODS}

\section{General Characteristics of Multistage Selection}

Several selection schemes were evaluated to illustrate the general characteristics of multistage selection compared with selection in a single step, i.e., after all information was available. In these schemes, selection was for one trait and in one sex only. In the first 2 sets of alternatives, the number of selection candidates and the total number of animals selected after the final selection step were fixed, i.e., the overall selected fraction ( $\mathrm{p}_{\mathrm{m}}$ for males, $\mathrm{p}_{\mathrm{f}}$ for females) was constant. Saxton (1983) showed that the response of multistage selection relative to the response of single-stage selection depends on the fractions selected in stage $1\left(\mathrm{p}_{1}\right)$ and in stage $2\left(\mathrm{p}_{2}\right)$, and on the ratio between the accuracies of the selection index in stage 1 and in stage 2. Therefore, fractions selected in the first and the second stage were varied, ranging from performing all selection after stage $2\left(p_{1}=1, p_{2}=p\right.$, with $p=$ overall selected fraction $)$ to all selection after stage $1\left(p_{1}=p, p_{2}=1\right)$. In all schemes, accuracy in the second stage was set to 0.93 , representing an index consisting primarily of information on a large group of progeny. In the first set of alternatives, the ratio of accuracies in stage 1 to stage 2 was set to 0.22 , reflecting a situation with relatively low accuracy of the stage 1 index (e.g., stage 1 consisting of pedigree information only). In a second set of alternatives this ratio was set to 0.69 , reflecting a situation with relatively high accuracy of the stage 1 index (e.g., stage 1 consisting of pedigree and own performance or sib information). Overall selected fraction (p) in both sets of schemes was either $0.01,0.02,0.05,0.10$, or 0.20 .

In a third set of alternatives, the selected fraction in the first stage $\left(\mathrm{p}_{1}\right)$ was varied from 0.01 to 1.0 , while keeping the number of selection candidates and the selected fraction in stage $2\left(\mathrm{p}_{2}\right)$ at a fixed level. Accuracies were equal to accuracies in the second set of alternatives. The third set of alternatives represented situations where the number of candidates available before selection in stage 1 increased with the intensity of first- 
stage selection. Selected fraction in stage 2 was 0.01 , $0.02,0.05,0.10$, or 0.20 .

Responses were computed using an exact method developed by Ducrocq and Colleau (1986), based on principles described by Tallis (1961) and Dutt (1973).

To translate some of the general characteristics of multistage selection to practical breeding schemes, dairy cattle breeding schemes utilizing MAS were simulated.

\section{Breeding Schemes Applying MAS}

The response in a number of schemes applying MAS preselection was compared with the response in a breeding scheme with preselection on pedigree information only. The comparison was carried out by deterministic simulation of multistage selection schemes, using the program SelAction (Rutten et al., 2002). This program predicts the rate of genetic gain based on advanced selection index theory. SelAction accounts for reduction in variance due to selection (Bulmer, 1971), and corrects selection intensities for finite population size and for the correlation between index values of family members (Meuwissen, 1991). Full pedigree information as with BLUP selection is accounted for (Villanueva et al., 1993), and the program is therefore an accurate approximation of stochastic simulation with BLUP. Deviations from normality after the first stage of selection are dealt with, and selection response is predicted for the equilibrium situation (Rutten et al., 2002), using the exact method of Tallis (1961) and Ducrocq and Colleau (1986). Features of SelAction and the theoretical background are described by Rutten and Bijma (2001a,b) and by Rutten et al. (2002).

Base breeding program. The breeding program was a closed nucleus scheme, with discrete generations. The breeding goal consisted of one trait, with heritability of 0.35 and phenotypic variance of 100 . Selection of males and females in stage 1 was for an index based on pedigree information only. In stage 2 , selection in females was for an index based on pedigree information and phenotypic information of the candidate and its half sibs and full sibs. In males, selection in stage 2 was for an index based on pedigree information, progeny information (100 daughters in the population), and sib information. This situation is comparable to selection for a milk production trait in dairy cattle. Each generation, 20 sires and 200 dams were selected to produce 4000 embryos. Half of these embryos resulted in live calves, with a sex ratio of 0.50 . In stage 1,200 males were selected out of 1000 to enter the progeny test. In stage 2, 20 males were selected out of 200 to produce the next generation. Selection in females reduced numbers from 1000 to 700 in stage 1 and from 700 to 200 in stage 2. Involuntary culling and death were ignored. The base breeding scheme is summarized in Figure 1.

Breeding schemes with MAS. In the breeding schemes with MAS, additional information, consisting of information on QTL, was available in the first stage. The QTL explained $5,10,20$, or $50 \%$ of the genetic variance, referred to as Q05, Q10, Q20, and Q50, respectively. The remaining genetic variation resulted from polygenes (i.e., not marked). In the simulations using SelAction, the QTL information was modeled as a trait that was correlated with the breeding goal trait and had a heritability of 1 . Information sources in the selection index were 1) QTL information, and 2) phenotype of the breeding goal trait, corrected for the QTL information. The correlations between the QTL and the breeding goal trait depended on the amount of variation that was explained by the QTL. The correlation between the QTL and the polygenic component was zero in the base generation (i.e., before selection). Genetic correlations of the breeding goal trait with the QTL and polygenic component were $\sqrt{q}$ and $\sqrt{1-q}$, respectively, where $q$ is the fraction of the genetic variance explained by the QTL. Phenotypic correlations of the breeding goal trait with the QTL and polygenic component were $\sqrt{q^{*} h_{0}^{2}}$ and $\sqrt{1-q^{*} h_{0}^{2}}$, respectively, where $h_{0}^{2}$ is the heritability of the overall trait. The heritability of the polygenic component was $\frac{1-q}{\frac{1}{h_{0}^{2}}-q}$.

Schemes were simulated with a fixed number of candidates, or with an increased number of candidates before preselection (relative to the base population).

Fixed number of candidates. The number of candidates before selection in stage 1 and the number of selected animals were fixed. Selection in stage 1 was on an index with information on parents (BLUP breeding values) and information on the QTL. In stage 2, information on sibs and own performance was added to the index for females, and information on sibs and progeny was added to the index for males.

Preselection in males. The level of preselection in bulls was varied from $\mathrm{p}_{1, \mathrm{~m}}=0.20$ (preselection equal to preselection in the base scheme) down to $\mathrm{p}_{1, \mathrm{~m}}=0.02$ (all selection in stage 1), with steps of 0.01 . In this way, the level of preselection where genetic progress was equal to genetic progress in the base scheme, could be determined. The selected fractions for the females in stage 1 and stage 2 were equal to selected fractions in the base scheme. For the scheme with all selection in stage $1\left(p_{1, m}=0.02\right)$, generation intervals were reduced, because there was no need to wait for progeny test 
Males

20 sires

个 $\mathrm{p}_{2, \mathrm{~m}}=0.10$

200 young bulls, progeny tested

$$
\text { 覀 } \mathrm{p}_{1, \mathrm{~m}}=0.20
$$

1000 born male calves

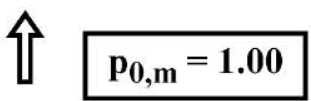

2000 male embryos produced

Information sources:

$p_{1, m}$ : pedigree information

$\mathbf{p}_{2, f}:$ pedigree + progeny + HS + FS
Females

200 dams

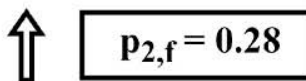

700 females with lactation information

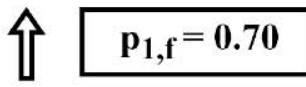

1000 born female calves

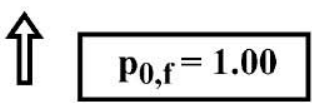

2000 female embryos produced

Information sources:

$p_{1, f}:$ pedigree information

Figure 1. Selection steps, selected fractions, and information sources for males and females in the base breeding scheme. Selected fractions are given as $\mathrm{p}_{\mathrm{a}, \mathrm{b}}$, with $\mathrm{a}=0,1$ or 2 , representing selection stage, and b equals $\mathrm{m}$ or $\mathrm{f}$, indicating males and females. There was no selection in embryos, indicated by $\mathrm{p}_{0, \mathrm{~m}}=\mathrm{p}_{0, \mathrm{f}}=1.00$.

results. In comparing results for this scheme with other schemes, genetic progress was corrected for the difference in generation intervals. The generation interval was assumed to be 3 in the scheme with $\mathrm{p}_{1, \mathrm{~m}}=0.02$, and 5 in all other schemes. Schemes are referred to as $\mathrm{M}_{\mathrm{Q} 05}, \mathrm{M}_{\mathrm{Q} 10}, \mathrm{M}_{\mathrm{Q} 20}$, and $\mathrm{M}_{\mathrm{Q} 50}$, where $\mathrm{M}$ indicates varying selection in males.

Preselection in females. The advantage of additional information is expected to be different for dams and sires. To quantify this difference, schemes were simulated where the level of preselection in females was varied from $p_{1, f}=0.70$ (equal to preselection in the base scheme) down to $\mathrm{p}_{1, \mathrm{f}}=0.20$ (all selection in the first stage), with steps of 0.05 . The selected fractions for the males in stage 1 and stage 2 were equal to the selected fractions in the base scheme. These schemes are referred to as $\mathrm{F}_{\mathrm{Q} 05}, \mathrm{~F}_{\mathrm{Q} 10}, \mathrm{~F}_{\mathrm{Q} 20}$, and $\mathrm{F}_{\mathrm{Q} 50}$, where $\mathrm{F}$ indicates varying selection in females.

Increased numbers of candidates. For the previously mentioned comparisons, the number of embryos (i.e., number of candidates before selection) was kept constant. However, application of genotyping techniques on embryos offers additional opportunities for preselection without increasing the number of transferred embryos. This practice was modeled by simulating breeding schemes with increased reproductive rates, i.e., 8000 instead of 4000 embryos were produced by 20 sires and 200 dams. The number of progeny tested bulls was kept constant. Therefore, selection in the first stage was more intense, i.e., $p_{1, m}=0.10$ for males, and $p_{1, f}=0.35$ for females. These schemes are referred to as $\mathrm{E}_{\mathrm{Q} 05}, \mathrm{E}_{\mathrm{Q} 10}, \mathrm{E}_{\mathrm{Q} 20}$, and $\mathrm{E}_{\mathrm{Q} 50}$, where $\mathrm{E}$ indicates that extra embryos were produced.

Overview. The general characteristics of the schemes are summarized in Table 1. Results of these schemes were compared with a corresponding base scheme with the same characteristics, but without a QTL. The value of the QTL information was assessed in 2 ways: 1) the increase in genetic gain; 2 ) the reduction in the number of sires required to be progeny tested to achieve the same rate of gain as in the base scheme.

\section{RESULTS}

\section{General Characteristics of Multistage Selection}

Figure 2 shows the response in multistage selection schemes with a high ratio between accuracies in stage 1 and stage 2 (i.e., a relatively high accuracy in stage 1 ). The response is expressed as a fraction of the response obtained when all selection was in stage $2\left(R_{0}\right)$, which is the maximum response. Note that the horizontal axis contains values for $1-p_{1}$, i.e., larger values indicate stronger selection in stage 1 .

Response was highest when there was no preselection. Depending on the overall selected proportion, there was only a slight reduction in response, even when considerable preselection was applied. The reduc- 
Table 1. Characteristics of simulated breeding schemes.

\begin{tabular}{|c|c|c|c|c|c|}
\hline \multirow[b]{2}{*}{ Scheme $^{1}$} & \multirow{2}{*}{$\begin{array}{l}\text { Number of } \\
\text { candidates } \\
\text { before } \\
\text { selection }^{2}\end{array}$} & \multicolumn{2}{|c|}{$\begin{array}{l}\text { Selected fractions, } \\
\text { males }^{3}\end{array}$} & \multicolumn{2}{|c|}{$\begin{array}{l}\text { Selected fractions, } \\
\text { females }^{3}\end{array}$} \\
\hline & & $\mathrm{p}_{1, \mathrm{~m}}$ & $\mathrm{p}_{\mathrm{m}}$ & $\mathrm{p}_{1, \mathrm{f}}$ & $\mathrm{p}_{\mathrm{f}}$ \\
\hline $\mathrm{M}_{\mathrm{Qxx}}$ & 1000 & $0.20-0.02$ & 0.02 & 0.70 & 0.20 \\
\hline $\mathrm{F}_{\mathrm{Qxx}}$ & 1000 & 0.20 & 0.02 & $0.70-0.20$ & 0.20 \\
\hline $\mathrm{E}_{\mathrm{Qxx}}$ & 2000 & $0.10-0.01$ & 0.01 & 0.35 & 0.10 \\
\hline
\end{tabular}

${ }^{1} \mathrm{Q}_{\mathrm{xx}}$ refers to the amount of genetic variance explained by the QTL, either $0,5,10,20$, or $50 \%$; $\mathrm{M}=$ varying preselection in males; $\mathrm{F}$ = varying preselection in females; $\mathrm{E}=$ increased embryo production.

${ }^{2}$ Equal for males and females; displayed are numbers per sex.

${ }^{3} \mathrm{p}_{1, \mathrm{~m}}=$ Selected fraction in the first stage, males; $\mathrm{p}_{\mathrm{m}}=$ overall selected fraction, males; $\mathrm{p}_{1, \mathrm{f}}=$ selected fraction in the first stage, females; $p_{\mathrm{f}}=$ overall selected fraction, females.

tion in response was due to culling of valuable animals that would have eventually been selected when all information became available. The reduction in response was small and approximately linear with increasing selection in stage 1 until a certain level of preselection, where response dropped at increasing rate. For stronger overall selection, more preselection could be applied before overall response dropped below $95 \%$ of the maximum response. For example, in Figure 2, when the overall selected fraction was 0.01 , response dropped below $95 \%$ when the selected fraction in stage 1 equaled $0.05\left(1-\mathrm{p}_{1}=0.95\right)$. This means that $95 \%$ of the candi- dates did not need to be retained until the second selection step, with only $5 \%$ loss in response. When the overall selected fraction was 0.20 , response dropped below $95 \%$ when the selected fraction in stage 1 equaled 0.42 $\left(1-p_{1}=0.58\right.$, Figure 2$)$.

The reduction in response depends on the ratio between accuracies of the selection index in stage 1 and stage 2 . This can be seen by comparing results in Figure 2 with results in Figure 3. The ratio of accuracies of the selection index in stage 1 and stage 2 was 0.69 in Figure 2 and 0.22 in Figure 3, respectively. In Figure 3 , a reduction of $5 \%$ of the response for overall selected

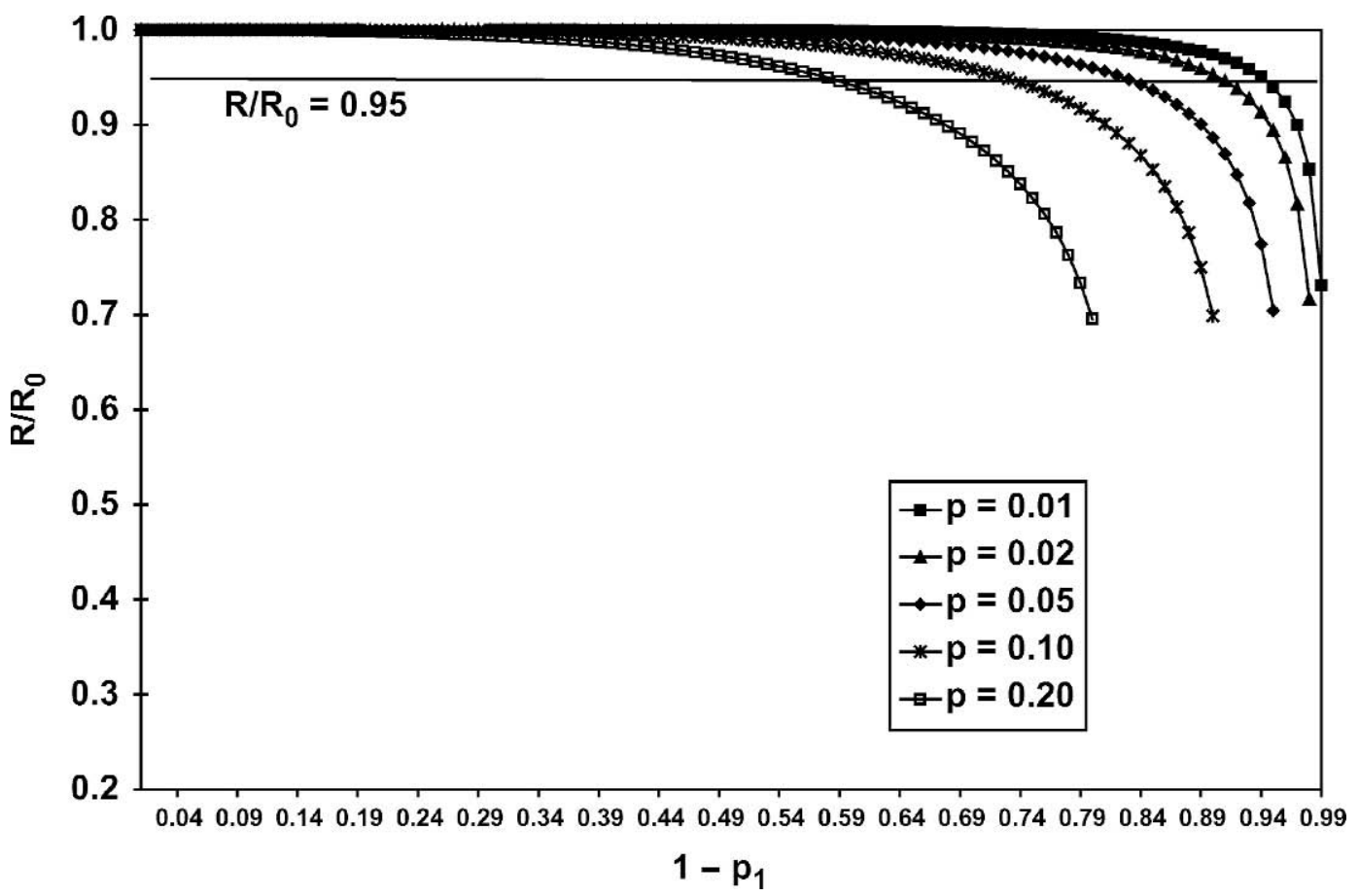

Figure 2. Response $(R)$ of schemes with different selected fractions in stage $1\left(p_{1}\right)$, expressed as fraction of response in a scheme with all selection in stage 2 , which is the maximum response $\left(\mathrm{R}_{0}\right)$. Responses are given for 5 different levels of overall selection (p), for high ratio (0.69) of accuracies of the index in stage 1 to stage 2 . Note that the horizontal axis contains values for $1-p_{1}$, i.e., larger values indicate stronger selection in stage 1 . The horizontal line $\left(R / R_{0}=0.95\right)$ indicates responses equal to $95 \%$ of the maximum response. 


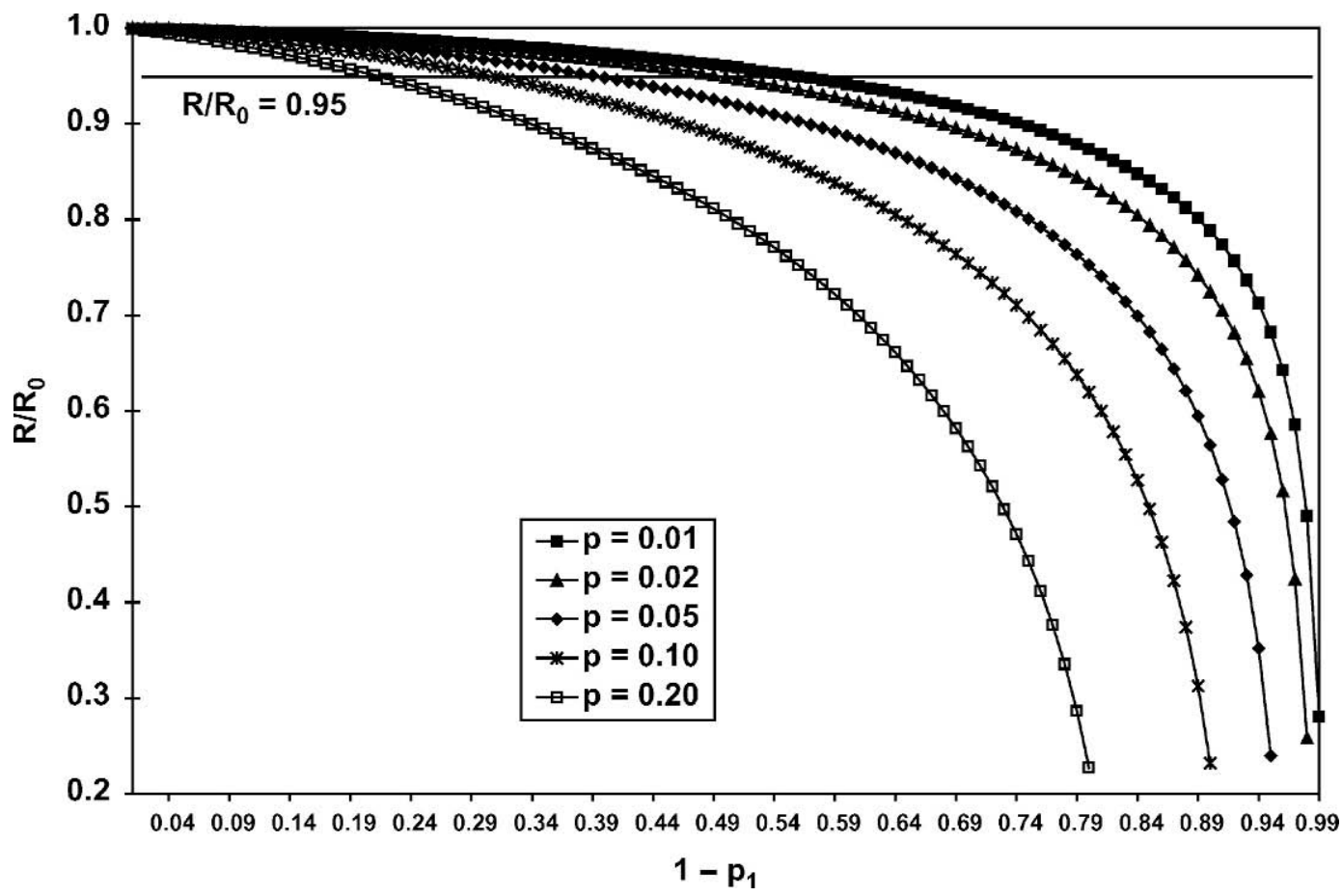

Figure 3. Response $(R)$ of schemes with different selected fractions in stage $1\left(p_{1}\right)$, expressed as fraction of response in a scheme with all selection in stage 2 , which is the maximum response $\left(R_{0}\right)$. Responses are given for 5 different levels of overall selection $(p)$, for low ratio $(0.22)$ of accuracies of the index in stage 1 to stage 2 . Note that the horizontal axis contains values for $1-p_{1}$, i.e., larger values indicate more selection in stage 1 . The horizontal line $\left(R / R_{0}=0.95\right)$ indicates responses equal to $95 \%$ of the maximum response.

fractions of 0.01 and 0.20 was obtained for selected fractions in stage 1 of $0.44\left(1-p_{1}=0.56\right)$ and 0.78 (1 $\left.-p_{1}=0.22\right)$, respectively. The difference between Figures 2 and 3 depends on the ratio of accuracies, and not on the absolute level of accuracies (Tallis, 1961).

In Figures 2 and 3, the selected fractions in stage 1 and stage 2 were varied, keeping the overall selected fraction constant. In Figure 4, selection in stage 2 was kept at the original level, and the number of selection candidates at the beginning of the selection process was increased. This resulted in increased overall selection intensity. When the selected fraction was 0.01 (selection in one stage), the increase in response was $8.5 \%$ when an additional preselection step was introduced with a selected fraction of 0.50 (Figure 4). For example, initially, 10 out of 1000 animals were selected in one stage. With preselection, 1000 out of 2000 animals were selected in stage 1 , and 10 out of 1000 in stage 2 . The increase in response was $24.1 \%$ when preselection with a selected fraction of 0.50 was introduced in a scheme with a selected fraction of 0.20 (Figure 4). Thus, when the selected fraction was already small, increase in response was relatively small when a preselection step was introduced. In the latter case (i.e., scheme with selected fraction 0.20 ), the increase in overall selection intensity was relatively higher than in the first case (scheme with selected fraction 0.01).

In conclusion, Figures 2 to 4 show that

1. Multistage selection leads to a decrease in response when compared with selection in one stage (Figures 2 and 3 ).

2. The reduction in response with increasing levels of preselection occurs at a slower rate when the overall selection intensity is higher (Figures 2 and 3).

3 . The reduction in response occurs at a slower rate when the accuracy in the first stage of selection is relatively high compared with the accuracy in the final stage of selection (comparing Figures 2 and 3).

4. Response from preselection on additional candidates increases with increasing selection intensity in the first stage (i.e., with increasing numbers of additional candidates), and the rate of this increase is greatest when the initial overall selection intensity is low (Figure 4).

5. The overall selection intensity and ratio of accuracies in the various stages of selection determine the "cost" of increasing selection intensity in the first stage. 


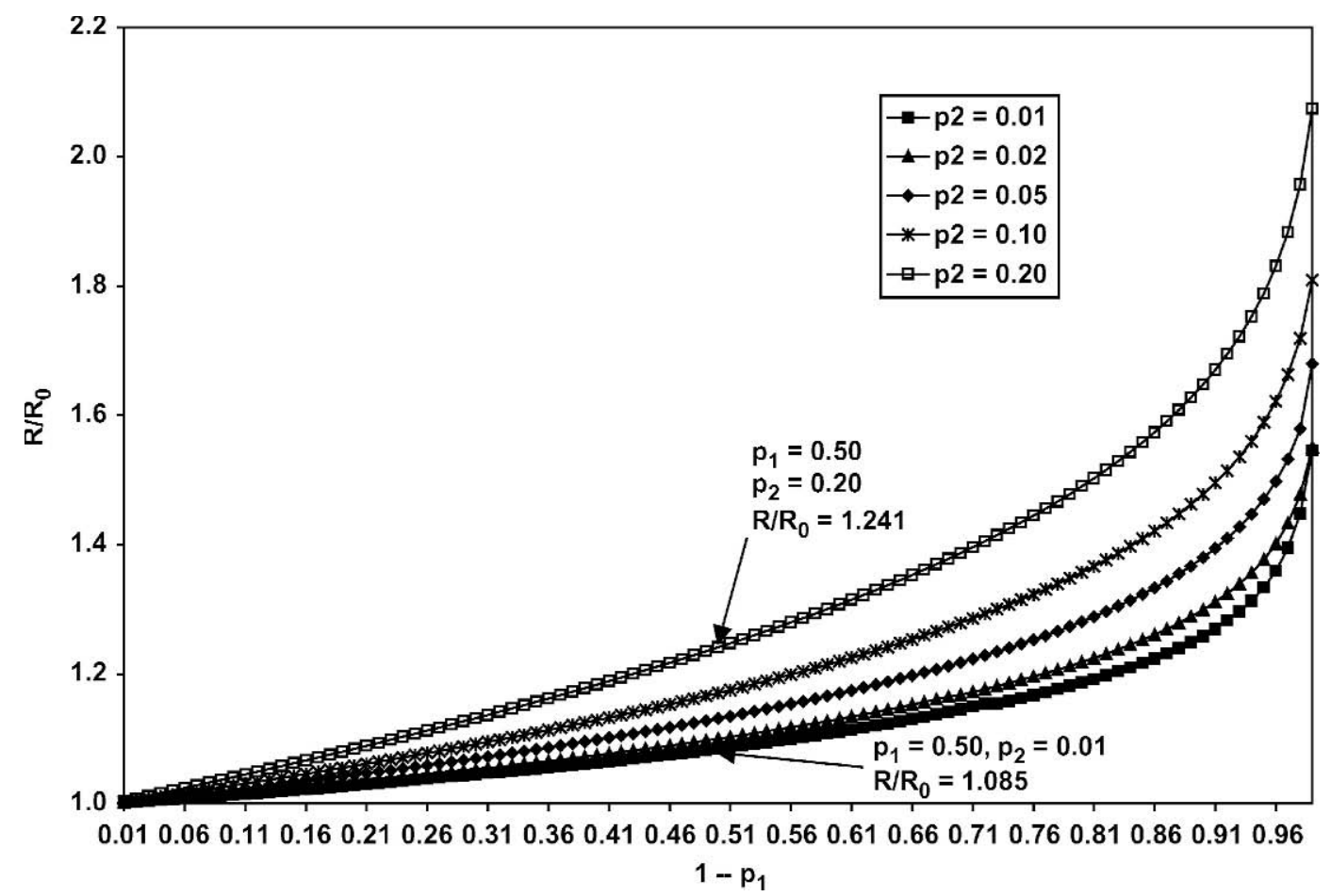

Figure 4. Response (R) of schemes with different selected fractions in stage $1\left(p_{1}\right)$, expressed as fraction of response in a scheme with all selection in stage 2 , which is the maximum response $\left(R_{0}\right)$. Responses are given for 5 different levels of selection in stage $2\left(p_{2}\right)$, for high ratio (0.69) of accuracies of the index in stage 1 to stage 2 . Compared with previous schemes, there are more selection candidates at the beginning. Note that the horizontal axis contains values for $1-p_{1}$, i.e., larger values indicate more selection in stage 1 .

\section{MAS Breeding Schemes with Varying Preselection in Males}

Figure 5 shows genetic response per generation relative to response in the base scheme for varying levels of preselection in bulls and various sizes of the QTL effect. Information on the QTL was available in both males and females, but preselection was applied only in the males. When the QTL explained 5\% of the genetic variance $\left(\mathrm{M}_{\mathrm{Q} 05}\right)$, genetic progress was $4.5 \%$ higher for selected fractions equal to that of the base scheme $\left(\mathrm{p}_{1, \mathrm{~m}}=0.20\right)$. For $\mathrm{M}_{\mathrm{Q} 10}, \mathrm{M}_{\mathrm{Q} 20}$, and $\mathrm{M}_{\mathrm{Q} 50}$, genetic response per generation was $7.7,12.5$, and $21.2 \%$ higher, respectively (Figure 5).

As can be seen in Figure 5, increasing the selection intensity in stage 1 while decreasing selection intensity in stage 2 led to decreased genetic progress. However, the decrease was small when halving the number of progeny tested animals (i.e., $\mathrm{p}_{1, \mathrm{~m}}$ decreased from 0.20 to 0.10 ), especially for large QTL. Genetic progress compared with the base scheme was $-3.9,+0.3,+6.7$, and $+19.0 \%$, for $\mathrm{M}_{\mathrm{Q} 05}, \mathrm{M}_{\mathrm{Q} 10}, \mathrm{M}_{\mathrm{Q} 20}$, and $\mathrm{M}_{\mathrm{Q} 50}$, respectively (Table 2, Figure 5), when the selected fraction in stage 1 was 0.10 . Generation interval in males and females was assumed to be 5 for all values of $\mathrm{p}_{1, \mathrm{~m}}$, except for $\mathrm{p}_{1, \mathrm{~m}}=0.02$, where the generation interval was assumed to be 3 . Due to the different generation interval when $\mathrm{p}_{1, \mathrm{~m}}=0.02$ (all selection in males was applied in stage 1, i.e., no progeny testing of young bulls was needed), these results deviated from the curve describing all other results (Figure 5). Responses for the schemes without progeny testing ranged from $-1.7 \%$ for $\mathrm{M}_{\mathrm{Q} 05}$ to $+68.3 \%$ for $\mathrm{M}_{\mathrm{Q} 50}$, relative to the base scheme.

The patterns of response for $\mathrm{M}_{\mathrm{Q} 50}$ and $\mathrm{M}_{\mathrm{Q} 05}$ were comparable to the patterns shown in Figure 2 and 3, respectively, for an overall selected fraction of 0.02 . For the schemes in Figure 2 and Figure 3, the ratios between accuracies in stage 1 and stage 2 were 0.69 and 0.22 , respectively. The ratio between accuracies in stage 1 and stage 2 was 0.72 for $\mathrm{M}_{\mathrm{Q} 50}$ and 0.31 for $\mathrm{M}_{\mathrm{Q} 05}$ (Table 2). Table 2 shows genetic progress at 2 levels of preselection in males $\left(\mathrm{p}_{1, \mathrm{~m}}=0.20\right.$ and $\left.\mathrm{p}_{1, \mathrm{~m}}=0.10\right)$. Also, the level of preselection resulting in responses equal to response in the base scheme is listed, and the contribution of females to genetic progress is shown.

For $\mathrm{M}_{\mathrm{Q} 05}$, genetic response was equal to response in the base scheme when the selected fraction in stage 1 was 0.13 (Table 2). This means that progeny testing 130 bulls in a MAS scheme with a QTL explaining 5\% of the genetic variance would yield genetic response equal to that of a nonMAS scheme where 200 bulls were 


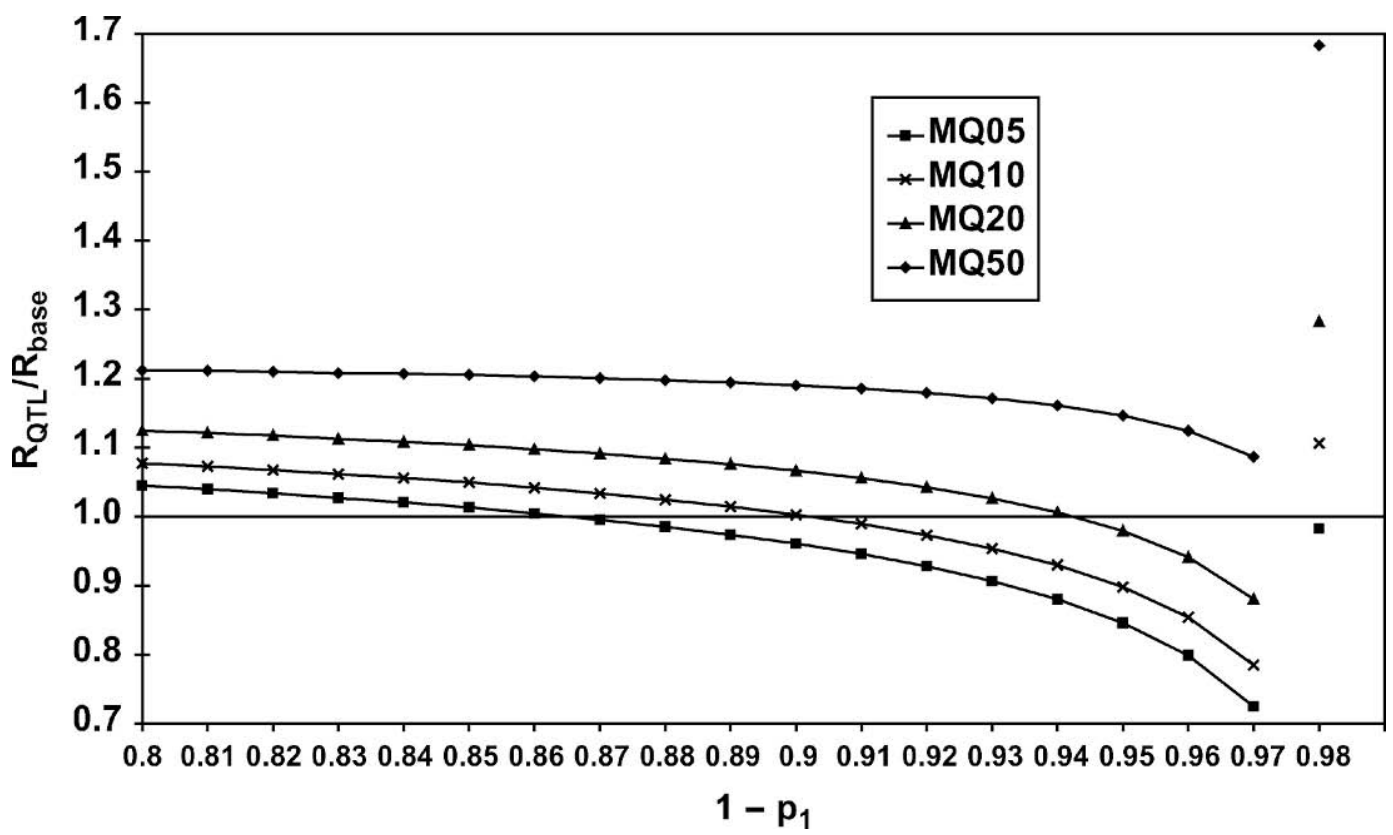

Figure 5. Genetic response per year relative to response in the base scheme, when information on a QTL explaining 5, 10, 20, or 50\% of the genetic variance was used. Selected fraction in males in stage 1 varied from 0.20 (equal to the base scheme; $1-p_{1, m}=0.80$ ) down to 0.02 . The different generation interval for $\mathrm{p}_{1, \mathrm{~m}}=0.02$ (no progeny testing) was accounted for. The number of selection candidates and overall selected fraction were constant and equal to the base scheme.

progeny tested. For $\mathrm{M}_{\mathrm{Q} 10}$ and $\mathrm{M}_{\mathrm{Q} 20}$, response was equal to response in the base scheme when selected fractions in stage 1 were approximately 0.10 and 0.06 , respectively, corresponding to 100 and 60 progeny tested bulls. Genetic response for $\mathrm{M}_{\mathrm{Q} 50}$ was always higher than genetic response in the base scheme.

In the base scheme, $28.0 \%$ of genetic progress was contributed by selection in females. When information on a QTL was used, the contribution of selection in females increased slightly. For $\mathrm{M}_{\mathrm{Q} 05}, \mathrm{M}_{\mathrm{Q} 10}, \mathrm{M}_{\mathrm{Q} 20}$, and $\mathrm{M}_{\mathrm{Q50}}$, the contribution of selection in females was 28.2, $28.5,29.1$, and $31.6 \%$ respectively, when selected fractions were equal to selected fractions in the base scheme. The addition of QTL information to the selection index led to increased accuracy of selection in stage 1 and stage 2 . The increase in accuracy in stage 1 was

Table 2. Genetic progress in marker-assisted selection (MAS) breeding schemes relative to base breeding scheme, for selected fraction in bulls in stage $1\left(\mathrm{p}_{1, \mathrm{~m}}\right)$ of 0.20 and 0.10 , selected fraction in stage 1 resulting in equal genetic progress as in the base scheme, and contribution of females to genetic progress.

\begin{tabular}{|c|c|c|c|c|c|}
\hline $\begin{array}{l}\text { MAS } \\
\text { scheme }^{1}\end{array}$ & $\begin{array}{l}\text { Ratio of } \\
\text { accuracies }^{2}\end{array}$ & $\begin{array}{l}\text { Genetic } \\
\text { progress, }{ }^{3} \\
p_{1, \mathrm{~m}}=0.20 \\
(\%)\end{array}$ & $\begin{array}{l}\text { Genetic } \\
\text { progress, }{ }^{3} \\
\mathrm{p}_{1, \mathrm{~m}}=0.10^{4} \\
(\%)\end{array}$ & $\begin{array}{l}\mathrm{p}_{1, \mathrm{~m}} \text { with } \\
\text { equal genetic } \\
\text { progress as in } \\
\text { base scheme }\end{array}$ & $\begin{array}{l}\text { Contribution } \\
\text { of females } \\
\text { to genetic } \\
\text { progress }^{5} \\
(\%)\end{array}$ \\
\hline $\mathrm{M}_{\mathrm{Q} 00}$ & 0.22 & 0 & -9.7 & 0.20 & 28.0 \\
\hline $\mathrm{M}_{\mathrm{Q} 05}$ & 0.31 & +4.5 & -3.9 & 0.13 & 28.2 \\
\hline $\mathrm{M}_{\mathrm{Q} 10}$ & 0.37 & +7.7 & +0.3 & 0.10 & 28.5 \\
\hline $\mathrm{M}_{\mathrm{Q} 20}$ & 0.48 & +12.5 & +6.7 & 0.06 & 29.1 \\
\hline $\mathrm{M}_{\mathrm{Q} 50}$ & 0.72 & +21.2 & +19.0 & 0.02 & 31.6 \\
\hline
\end{tabular}

${ }^{1}$ MAS scheme: M refers to varying marker-assisted preselection in males, Qxx indicates percentage of additive genetic variance explained by the QTL.

${ }^{2}$ Ratio of accuracies in stage 1 and stage 2

${ }^{3}$ Relative to base breeding scheme.

${ }^{4}$ This corresponds to a scheme where the number of progeny-tested bulls was only $50 \%$ of the number of progeny-tested bulls in the base scheme.

${ }^{5}$ With selected fraction in stage 1 equal to selected fraction in stage 1 in the base scheme; in the base scheme, selection was on pedigree information only. 


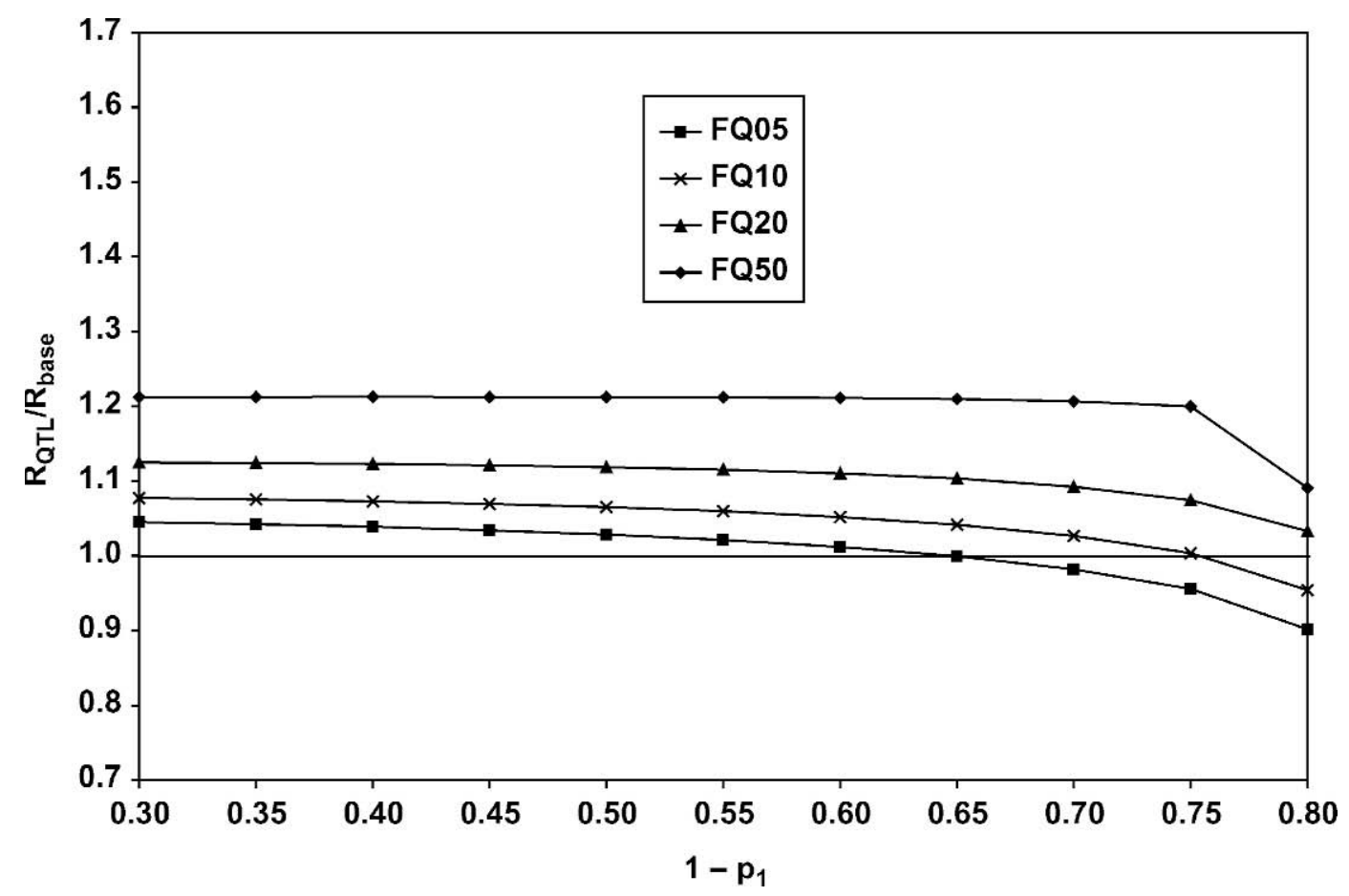

Figure 6. Genetic response per generation relative to response in the base scheme (no QTL), when information on a QTL explaining 5, 10,20 , or $50 \%$ of the genetic variance was used. Selected fraction in females in stage 1 varied from 0.70 (equal to the base scheme; 1 $\mathrm{p}_{1, \mathrm{f}}=0.30$ ) down to 0.20 . Overall selection and selection in males was constant and equal to selection in the base scheme.

equal for males and females, whereas the increase in accuracy in stage 2 was relatively higher for females than for males. This resulted in a relatively higher contribution of females to the genetic progress for schemes with a QTL explaining a higher percentage of the additive genetic variance.

\section{MAS Breeding Schemes with Varying Preselection in Females}

Figure 6 shows genetic response relative to the base scheme when preselection in females was varied while keeping the selected fraction in stage 1 of males at 0.20 . Information on the QTL was available in both males and females, but preselection was applied only in the females. This represents breeding schemes where preselection leads to fewer females that need to be performance tested, to reduce the size of the nucleus and reduce the costs of recording. Curves are relatively flat, which indicates that genetic response was only slightly decreased with increased preselection of females. The ratio between accuracies in stage 1 and stage 2 was relatively high, ranging from 0.50 for alternative $\mathrm{F}_{\mathrm{Q} 05}$ to 0.91 for alternative $\mathrm{F}_{\mathrm{Q} 50}$. Consequently, a relatively flat curve was expected, based on the general characteristics of multistage selection. With a selected fraction in stage 1 of 0.35 instead of 0.70 (i.e., halving the size of the nucleus), the genetic progress compared with the base scheme was $-0.1,+4.2,+10.3$, and $+21.0 \%$, for $\mathrm{F}_{\mathrm{Q} 05}$, $\mathrm{F}_{\mathrm{Q} 10}, \mathrm{~F}_{\mathrm{Q} 20}$, and $\mathrm{F}_{\mathrm{Q} 50}$, respectively. When the selected fraction in stage 1 of males was 0.10 instead of 0.20 , curves looked similar, but genetic progress was at a lower level. When $\mathrm{p}_{1, \mathrm{f}}$ in females was 0.35 , the genetic progress compared with the base scheme was -8.5, -3.3, +4.5 , and $+18.8 \%$, for $\mathrm{F}_{\mathrm{Q} 05}, \mathrm{~F}_{\mathrm{Q} 10}, \mathrm{~F}_{\mathrm{Q} 20}$, and $\mathrm{F}_{\mathrm{Q} 50}$, respectively.

\section{Increased Embryo Production}

We evaluated the consequences of doubling the number of embryos produced (from 4000 to 8000 ) by the same number of parents. In these schemes, selection in stage 1 was more intense, with $\mathrm{p}_{1, \mathrm{~m}}$ and $\mathrm{p}_{1, \mathrm{f}}$ equal to 0.10 and 0.35 , respectively. Genetic progress was 12.1 , 17.1, 24.5, and $37.6 \%$ higher than in the base scheme with 4000 embryos, for $\mathrm{E}_{\mathrm{Q} 05}, \mathrm{E}_{\mathrm{Q} 10}, \mathrm{E}_{\mathrm{Q} 20}$, and $\mathrm{E}_{\mathrm{Q} 50}$, respectively (Table 3 ).

Part of the additional genetic progress in these breeding schemes was caused by increased production of embryos. Therefore, these schemes were also compared with a base scheme with increased embryo production and increased preselection $\left(\mathrm{p}_{1, \mathrm{~m}}=0.10\right)$. Doubling the number of embryos increased genetic progress by $4.8 \%$ in the base scheme. This means, for example, that the 
Table 3. Genetic progress in marker-assisted selection (MAS) breeding schemes relative to base breeding scheme and alternative base breeding scheme with increased embryo production, for selected fraction in bulls in stage $1\left(\mathrm{p}_{1, \mathrm{~m}}\right)$ of 0.10 and 0.05 , selected fraction in stage 1 resulting in equal genetic progress as in the alternative base scheme, and contribution of females to genetic progress.

\begin{tabular}{|c|c|c|c|c|c|}
\hline $\begin{array}{l}\text { MAS } \\
\text { scheme }^{1}\end{array}$ & $\begin{array}{l}\text { Genetic } \\
\text { progress, }{ }^{2} \\
\mathrm{p}_{1, \mathrm{~m}}=0.10 \\
(\%)\end{array}$ & $\begin{array}{l}\text { Genetic } \\
\text { progress, }{ }^{3} \\
\mathrm{p}_{1, \mathrm{~m}}=0.10 \\
(\%)\end{array}$ & $\begin{array}{l}\text { Genetic } \\
\text { progress, } \\
\mathrm{p}_{1, \mathrm{~m}}=0.05^{4} \\
(\%)\end{array}$ & $\begin{array}{l}\mathrm{p}_{1, \mathrm{~m}} \text { with } \\
\text { equal genetic } \\
\text { progress as in } \\
\text { alternative } \\
\text { base scheme }\end{array}$ & $\begin{array}{l}\text { Contribution } \\
\text { of females } \\
\text { to genetic } \\
\text { progress }^{5} \\
(\%)\end{array}$ \\
\hline $\mathrm{E}_{\mathrm{Q} 00}$ & 0 & 0 & -9.7 & 0.10 & 29.8 \\
\hline $\mathrm{E}_{\mathrm{Q} 05}$ & +12.1 & +7.0 & -1.6 & 0.06 & 30.6 \\
\hline $\mathrm{E}_{\mathrm{Q} 10}$ & +17.1 & +11.8 & +4.1 & 0.04 & 31.1 \\
\hline $\mathrm{E}_{\mathrm{Q} 20}$ & +24.5 & +18.9 & +12.6 & 0.02 & 32.0 \\
\hline $\mathrm{E}_{\mathrm{Q} 50}$ & +37.6 & +31.3 & +28.7 & $\ldots$ & 34.6 \\
\hline
\end{tabular}

${ }^{1}$ MAS scheme: E refers to marker-assisted preselection in embryos, Qxx indicates percentage of additive genetic variance explained by the QTL.

${ }^{2}$ Relative to base breeding scheme with $\mathrm{p}_{1, \mathrm{~m}}=0.20$.

${ }^{3}$ Relative to alternative base breeding scheme with increased embryo production, with $\mathrm{p}_{1, \mathrm{~m}}=0.10$.

${ }^{4}$ This corresponds to a scheme where the number of progeny-tested bulls was only $50 \%$ of the number of progeny-tested bulls in the base scheme.

${ }^{5}$ With selected fraction in stage 1 equal to selected fraction in stage 1 in the base scheme; in the base scheme, selection was on pedigree information only.

increased genetic gain of $12.1 \%$ of $\mathrm{E}_{\mathrm{Q} 05}$ consisted of a $4.8 \%$ increase from doubling the number of embryos and a $7.0 \%$ increase due to the application of MAS (Table 3). To obtain responses equal to a base scheme with $\mathrm{p}_{1, \mathrm{~m}}=0.10$, the number of progeny-tested young bulls could be reduced from 200 in the base scheme to 120 , 80, or 40 in schemes $\mathrm{E}_{\mathrm{Q} 05}, \mathrm{E}_{\mathrm{Q} 10}$, and $\mathrm{E}_{\mathrm{Q} 20}$, respectively.

\section{DISCUSSION}

\section{Simulation Model}

In this study, breeding schemes for dairy cattle when QTL information was available were compared by deterministic simulation of multistage selection schemes using the program SelAction (Rutten et al., 2002). A QTL explaining $5 \%$ of the genetic variance could be used to increase genetic gain by $4.5 \%$ or to reduce the number of progeny-tested bulls by $35 \%$ while maintaining the same rate of genetic gain. In schemes utilizing QTL that explained up to $50 \%$ of the genetic variance, or utilizing QTL information on embryos, higher increases of genetic response (up to $+31.3 \%$ ) and further reduction of the number of progeny-tested bulls (to no progeny testing at all) could be realized.

Information sources for the index in stage 1 were included in the index for stage 2 as well, resulting in a correlation between index 1 and 2 . This correlation is equal to the ratio of the accuracies of index 1 and index 2 (Saxton, 1983). Xie and Xu (1998) compared the efficiency of multistage MAS with efficiency of conventional phenotypic selection. They applied a similar procedure, but to avoid numerical multiple integration, they imposed a constraint on the solutions for multistage MAS by forcing the covariance between the index in stage 1 and stage 2 to be 0 . This has an effect similar to that of a restricted index, resulting in less than optimal gain. They did not account for the effect of finite population size on selection intensities and for reduction in variance due to selection. The program SelAction (used here) accounts for these effects, and constructs an optimum index using full pedigree information with an animal model (Villanueva et al., 1993).

Schemes for MAS tend to have high responses initially, due to strong emphasis on marked genetic variance (e.g., Spelman and Garrick, 1997). Depending on initial allele frequencies, the effect of the QTL, and selection intensity on the QTL, the variation due to the QTL decreases after the initial generations of MAS but decreases at a slower rate in later generations. This reduction in QTL variance leads to decreasing marginal response over the base scheme without QTL information across time. In our study, we calculated equilibrium rates of response, assuming an infinitesimal model (or a relatively large number of small QTL). Therefore, it was not possible to evaluate response on a per year basis, and to look at differences between short-term response and long-term response, as discussed by Gibson (1994). The response shortly after the implementation of MAS was not predicted, but this response is expected to be higher than the equilibrium response presented here. According to this line of reasoning, response in later generations may be overestimated, unless new QTL are detected continuously.

In this study, the QTL was modeled as a trait correlated with the breeding goal trait. We assumed that the 
QTL behaves as a polygenic trait that remains normally distributed over time. This assumption is probably only valid if the QTL is, in fact, not one QTL, but several unlinked QTL, where each QTL has multiple alleles. The continuous detection of new QTL over time would make the assumption more realistic, because it would allow for a certain fraction of the genetic variance to be explained by QTL at any point in time.

The simulation model ignores inbreeding (Rutten et al., 2002). Ideally, schemes with equal rates of inbreeding should be compared. The rate of inbreeding is proportional to the square of the long-term genetic contributions of selected animals. This latter term is likely to differ between the schemes evaluated in this study, resulting in different rates of inbreeding. It is a considerable challenge, however, to determine the expected rate of inbreeding for multistage selection schemes that are simulated deterministically.

\section{Genetic Response in MAS Schemes}

Several authors have studied MAS schemes for dairy cattle. Responses from MAS obtained in these studies varied widely, depending on size of the QTL, type of breeding scheme, and the way of calculating response, e.g., equilibrium rates of response or response from one round of selection, response in young bulls or response in population females. In the following section, results from a number of MAS-studies that looked at preselection will be discussed.

The use of markers to select young bulls was first suggested by Soller and Beckmann (1982). Extra gain in their scheme was estimated (Smith and Simpson, 1986) at $46 \%$ for direct selection on 20 QTL explaining $50 \%$ of the genetic variance. Kashi et al. (1990) reported an increase of up to $26 \%$ in genetic merit of bulls entering the progeny test, resulting from one round of marker-assisted selection of candidate bulls before entering the progeny test. In this case, selection was based on haplotypes of polyallelic markers associated with 20 segregating QTL explaining all the genetic variance. They did not account for the loss in genetic variation as a result of MAS. Mackinnon and Georges (1998) reevaluated the approach by Kashi et al. (1990). They found an $8 \%$ increase in response in young bulls from one round of selection in a bottom-up scheme when the QTL explained 10\% of the genetic variance. For a QTL explaining $50 \%$ of the genetic variance, increase in response was $23 \%$. In the current study, equilibrium response increased by $7.7 \%$ and $21.2 \%$ for QTL explaining $10 \%$ and $50 \%$ of the genetic variance, respectively, which is in the same range as the response obtained by Mackinnon and Georges (1998). Spelman and Garrick (1998) found a 9\% increase in rate of gain from one round of MAS in a combination of a top-down and a bottom-up scheme (Mackinnon and Georges, 1998). The QTL explained $16.7 \%$ of the genetic variance, and bull dams produced 40 progeny. Only one bull per full-sib group was selected. The QTL information was used on both the dam and the sire selection paths, in a population that had been selected for 1 to 2 generations. In the current study, 5 male progeny were produced per bull dam and selection was across families, not distinguishing between full sibs and unrelated animals. When the QTL explained 10 or $20 \%$ of the genetic variance, equilibrium response increased by 7.7 and $12.5 \%$, respectively, which is comparable to the response observed by Spelman and Garrick (1998).

\section{Implications for the Breeding Program}

Results in Figures 5 and 6 indicate, that increased accuracy of selection in stage 1 facilitates a reduction of the number of animals entering into progeny or performance testing. For example, a QTL explaining 5\% of the genetic variance would allow a reduction from 200 to 130 progeny-tested young bulls without affecting the genetic response. Doubling the number of embryos produced in combination with preselection for a QTL explaining $5 \%$ of the genetic variance would require the progeny testing of only 80 young bulls to obtain the genetic response of the base breeding scheme. Figure 5 also shows that schemes without progeny testing would be more beneficial than the base breeding scheme for QTL explaining from approximately $6 \%$ of the genetic variance and over, especially due to the huge decrease in the generation interval from 5 to 3 units.

In the studied alternatives, the intensity of preselection was increased in one of the sexes to look at differences between the effects of increased preselection in either sex. However, increased preselection may be applied in both sexes. Additional calculations (results not shown) on a few situations with increased preselection in both sexes were carried out, to look at implications for the potential reduction in progeny-tested bulls. For example, when the selected fraction for females in stage 1 was 0.40 instead of 0.70 , the number of progenytested bulls could be reduced only to 180 instead of 130 , to have the same genetic progress as in the base scheme.

These examples demonstrate that QTL information can be used in various ways, and the costs and benefits differ accordingly. Depending on which scheme is adopted, a scheme utilizing QTL information will require additional investment for production of extra embryos, collection of DNA, genotyping of embryos or newborn animals and their relatives, etc. Cost savings can be made with regard to cost for collection of information on progeny test daughters (labor, incentives paid to 
farmers), variable cost for buying, feeding, and housing of young and waiting bulls, fixed cost for housing (when the number of progeny-tested bulls is reduced substantially, less housing facilities are needed), etc. The overall selection intensity and ratio of accuracies in the various stages of selection determine the "cost" of increasing selection intensity in the first stage, as was concluded from the study on the general characteristics of multistage selection. The question of whether it is beneficial to implement MAS or not depends on the net result of additional cost, savings, and benefits from increased genetic progress of the chosen breeding program.

The QTL information can be measured accurately on newborn animals or on embryos (Bredbacka, 2001), which offers good opportunities for preselection and reduction of the number of progeny-tested or performance-tested animals by MAS. Schemes with increased selection intensity for males in stage 1 seem most promising, because reducing the number of progeny-tested young bulls can reduce the cost of the breeding program considerably. Comparing Figure 6 with Figure 5, a relatively higher reduction in the number of performancetested females would be possible, but this is likely to have less impact on cost reduction.

Breeding goals in dairy cattle consist of a variety of traits. Although QTL have been mapped for quite a few of these traits, in some cases explaining approximately $50 \%$ of the genetic variation of a particular trait (e.g., Grisart et al., 2002), the fraction of the variation of the overall breeding goal explained by QTL is likely to be moderate. For breeding schemes in the current study, the fraction of the genetic variance in the breeding goal explained by the QTL was assumed to be 5\% or higher. Even for the QTL explaining 5\% of the genetic variance, there was a considerable impact of marker-assisted selection on the genetic response.

\section{CONCLUSIONS}

For reasons mainly related to cost, current dairy cattle breeding programs are set up as multistage breeding programs, even though response to multistage selection is reduced compared with response to single-stage selection. However, even for schemes with considerable emphasis on selection in the first stage, the reduction in response is relatively low when accuracy of selection in stage 1 is relatively high, compared with accuracy of selection in the final stage. The availability of QTL information results in higher accuracies in stage 1 . Incorporation of QTL information in multistage dairy cattle breeding programs may have considerable impact on genetic progress and the structure of the breeding program. In the presented alternative breeding pro- grams, additional genetic progress ranged from $4.5 \%$ (QTL information on newborn animals, QTL explaining $5 \%$ of the genetic variance) to $31.3 \%$ (QTL information on embryos, QTL explaining 50\% of the genetic variance). The availability of QTL information early in life allowed for higher selection intensity in the first stage of selection, resulting in fewer progeny-tested bulls (up to 35 and $100 \%$ reduction in the mentioned schemes, respectively), while maintaining genetic progress at the same or higher levels as the base scheme without QTL information. The challenge for breeding organizations is to find the optimum breeding program with regard to additional genetic progress and additional (or reduced) cost, by adapting the size of the breeding program while incorporating QTL information.

\section{ACKNOWLEDGMENTS}

We thank Sijne van der Beek and Marco Bink for their contribution to the discussions on this work. We appreciate the financial support by Holland Genetics and by Senter, division of the Dutch Ministry of Economic Affairs (grant BTS-98194).

\section{REFERENCES}

Ashwell, M. S., C. E. Rexroad, Jr., R. H. Miller, and P. M. VanRaden. 1996. Mapping economic trait loci for somatic cell score in Holstein cattle using microsatellite markers and selective genotyping. Anim. Genet. 27:235-242.

Blott, S., J. J. Kim, S. Moisio, A. Schmidt-Kuntzel, A. Cornet, P. Berzi, N. Cambisano, C. Ford, B. Grisart, D. Johnson, L. Karim, P. Simon, R. Snell, R. Spelman, J. Wong, J. Vilkki, M. Georges, F. Farnir, and W. Coppieters. 2003. Molecular dissection of a quantitative trait locus. A phenylalanine-to-tyrosine substitution in the transmembrane domain of the bovine growth hormone receptor is associated with a major effect on milk yield and composition. Genetics 163:253-266.

Bredbacka, P. 2001. Progress on methods of gene detection in preimplantation embryos. Theriogenology 55:23-34.

Bulmer, M. G. 1971. The effect of selection on genetic variability. Am. Nat. 105:201-211.

Ducrocq, V., and J. J. Colleau. 1986. Interest in quantitative genetics of Dutt's and Deak's methods for numerical computation of multivariate normal probability integrals. Genet. Sel. Evol. 18:447474.

Dutt, J. E. 1973. A representation of multivariate probability integrals by integral transforms. Biometrika 60:637-645.

Georges, M., D. Nielsen, M. Mackinnon, A. Mishra, R. Okimoto, A. T. Pasquino, L. S. Sargeant, A. Sorensen, M. R. Steele, X. Zhao, J. E. Womack, and I. Hoeschele. 1995. Mapping quantitative trait loci controlling milk production in dairy cattle by exploiting progeny testing. Genetics 139:907-920.

Gibson, J. P. 1994. Short-term gain at the expense of long-term response with selection of identified loci. Proc. 5th World Congr. Genet. Appl. Livest. Prod., Guelph, Canada 21:201-204.

Grisart, B., W. Coppieters, F. Farnir, L. Karim, C. Ford, P. Berzi, N. Cambisano, M. Mni, S. Reid, P. Simon, R. Spelman, M. Georges, and R. Snell. 2002. Positional candidate cloning of a QTL in dairy cattle: Identification of a missense mutation in the bovine DGAT1 gene with major effect on milk yield and composition. Genome Res. 12:222-231. 
Kashi, Y., E. Hallerman, and M. Soller. 1990. Marker-assisted selection of candidate bulls for progeny testing programmes. Anim. Prod. 51:63-74.

Mackinnon, M. J., and M. A. J. Georges. 1998. Marker-assisted preselection of young dairy sires prior to progeny-testing. Livest. Prod. Sci. 54:229-250.

Meuwissen, T. H. E. 1991. Reduction of selection differentials in finite populations with a nested full-half sib family structure. Biometrics 47:195-203.

Meuwissen, T. H. E., and M. E. Goddard. 1996. The use of marker haplotypes in animal breeding schemes. Genet. Sel. Evol. 28:161-176.

Meuwissen, T. H. E., and J. A. M. van Arendonk. 1992. Potential improvements in rate of genetic gain from marker-assisted selection in dairy cattle breeding schemes. J. Dairy Sci. 75:1651-1659.

Rutten, M. J. M., and P. Bijma. 2001a. SelAction - Description of the program. http://www.zod.wau.nl/abg/selaction/resource/description.pdf. Accessed Oct. 1, 2004.

Rutten, M. J. M., and P. Bijma. 2001b. SelAction - Manual. http:// www.zod.wau.nl/abg/selaction/resource/manual.pdf. Accessed Oct. 1, 2004

Rutten, M. J. M., P. Bijma, J. A. Woolliams, and J. A. M. van Arendonk. 2002. SelAction: Software to predict selection response and rate of inbreeding in livestock breeding programs. J. Hered. 93:456-458.

Saxton, A. M. 1983. A comparison of exact and sequential methods in multistage selection. Theor. Appl. Gen. 66:23-28.
Shuster, D. E., M. E. Kehrli, Jr., M. R. Ackermann, and R. O. Gilbert. 1992. Identification and prevalence of a genetic defect that causes leukocyte adhesion deficiency in Holstein cattle. Proc. Natl. Acad. Sci. USA 89:9225-9229.

Smith, C., and S. P. Simpson. 1986. The use of genetic polymorphisms in livestock improvement. J. Anim. Breed. Genet. 103:205-217.

Soller, M., and J. S. Beckmann. 1982. Restriction fragment length polymorphisms and genetic improvement. Proc. 2nd World Congr. Genet. Appl. Livest. Prod., Madrid, Spain VI:396-404.

Spelman, R. J., and D. J. Garrick. 1997. Utilisation of marker assisted selection in a commercial dairy cow population. Livest. Prod. Sci. 47:139-147.

Spelman, R. J., and D. J. Garrick. 1998. Genetic and economic responses for within-family marker-assisted selection in dairy cattle breeding schemes. J. Dairy Sci. 81:2942-2950.

Spelman, R. J., D. J. Garrick, and J. A. M. van Arendonk. 1999 Utilisation of genetic variation by marker-assisted selection in commercial dairy cattle populations. Livest. Prod. Sci. 59:51-60.

Tallis, G. M. 1961. The moment generating function of the truncated multi-normal distribution. J. R. Stat. Soc. B 23:223-229.

Villanueva, B., N. R. Wray, and R. Thompson. 1993. Prediction of asymptotic rates of response from selection on multiple traits using univariate and multivariate best linear unbiased predictors. Anim. Prod. 57:1-13.

Xie, C., and S. Xu. 1998. Efficiency of multistage marker-assisted selection in the improvement of multiple quantitative traits. Heredity 80:489-498. 